\title{
Ground moving target indication and parameters estimation using a dual-frequency synthetic aperture radar
}

\author{
Gaohuan Lv*, Junfeng Wang, Xingzhao Liu and Kaizhi Wang
}

\begin{abstract}
A new scheme is presented to estimate the range and azimuth velocity components of a detected moving target by using a dual-frequency synthetic aperture radar (SAR). It consists of a moving target detector, a range velocity estimator, and an azimuth velocity estimator. In this scheme, two original SAR images are achieved from the returns first, and then processed by a symmetric defocusing filter pair (SDFP) to produce two defocused images. By comparing the sharpness of the two defocused images, the moving targets are indicated and isolated form each original SAR image. For a selected moving target, its range velocity component is estimated by using a Doppler ambiguity solver and a stepped approximation-and-comparison algorithm. After range velocity compensated, the target in the patch is concentrated in less range bins, and its azimuth velocity component is estimated by using an SDFP bank. Finally, the moving target is refocused and its azimuth displacement caused by range velocity component is corrected. The effectiveness of the proposed scheme is confirmed by the experiments with the field and simulated data.
\end{abstract}

\section{Introduction}

Synthetic aperture radar (SAR) has been widely used in many civilian and military applications, and the SAR with ground moving targets indication (GMTI) is a very hot topic in recent years. As many literatures discussed, if the returns from a moving target are processed in the same way as the stationary returns, the target will appear as an azimuth shift due to the range motion, and the image of the target will be smeared in the azimuth direction due to the azimuth motion [1]. Moving target detection and velocity components estimation are the two main tasks of GMTI in SAR $[2,3]$. As detection methods are well-developed in many literatures, we will focus on the algorithms about estimation of velocity components.

Conventional moving target indicators adopt multiantennae technique, and they generally require a calibrated and time-invariant radar system. In practice, the system is so complex that both high hardware and computation efforts are needed in implementation [3-10]. Recently many GMTI methodologies based on a single antenna SAR or a single complex-valued SAR image,

${ }^{*}$ Correspondence: Ivgh@sjtu.edu.cn

Department of Electronic Engineering, Shanghai Jiao Tong University, Dongchuan Road, Shanghai, PRC e.g., auto-focusing [11], antenna beam patten transforming [12], and SAR stacks [13], were developed and got many effective results. However, the proposed methods suffer from either a high computation effort or unsatisfactory estimate accuracy under the condition of high signal-to-clutter-plus-interference-ratio.

It is known that the moving-target-originated azimuth phase history is characterized only by the Doppler shift and the Doppler rate [14,15]. As the Doppler shift is aliased by the sampling of the pulse repetition frequency (PRF), it can be considered to be made up of an "integer PRF part" (or Doppler ambiguity) and a "fractional PRF part" (or baseband Doppler centroid). Many Doppler ambiguity solver (DAS) algorithms, such as multi-PRF [16], wavelength diversity [17], multi-look cross correlation, multi-look beat frequency algorithms [18], have been developed to estimate the two parts based on a correlation and regression procedure in the time or the frequency domain $[19,20]$. However, these methods aim to estimate the squint angle originally, and the estimators will be stranded when the target is submerged in the clutter or dispersed in many range bins. They cannot be used to estimate range velocity of a moving target directly. An algorithm called reflectivity displacement method [21,22]

\section{Springer}

(c) 2012 Lv et al: licensee Springer. This is an Open Access article distributed under the terms of the Creative Commons

Attribution License (http://creativecommons.org/licenses/by/2.0), which permits unrestricted use, distribution, and reproduction in any medium, provided the original work is properly cited. 
is proposed to solve this problem. The method is based on the analysis of the azimuth spectrum of the radar raw data and the primary condition for implementation is the use of a wide azimuth antenna beam, and the accuracy of the method depends on the reflectivity of the target. Rüegg et al. use a dual frequency millimeter wave SAR with mono-pulse processing for GMTI [21]. In this method, a baseband chirp signal is carried by two different frequencies, i.e., 35 and $96 \mathrm{GHz}$. Two SAR images are achieved from the two kinds of returns simultaneously, and mono-pulse ratio is chosen as a feature value to detect moving targets and estimate the velocity components. This method demonstrates good detection performance for slow-moving targets, but if a target has a high azimuth velocity and a small radar cross section as a car on a freeway does, it may disappear. A newer DAS based on the range alignment method was proposed by Wang et al. [23]. It is based on the fact that range migration can be corrected by shifting the Doppler slices such that their envelopes are similar, and thus the Doppler centroid can be estimated from the shifting step. This method also suffers from the weak reflectivity of the moving target and strong background.

In this article, an effective GMTI scheme is proposed based on a single antenna SAR using a dual-frequency chirp waveform. The scheme detects moving targets by using symmetric defocusing filter pairs (SDFP). It estimates range velocity component of a moving target by using a new DAS model and a stepped approximationand-comparison (SAC) algorithm, and estimates the azimuth velocity component by using an SDFP bank.

An SDFP processes a complex-valued SAR image to generate two defocused SAR images. In the two defocused images, the background is smeared to the same extent, but the moving targets are defocused differently. By comparing the sharpness of the two defocused SAR images patch by patch, the moving targets can be indicated adaptively and automatically.

The detected moving targets are cropped from the two original SAR images and transformed to range Doppler domain. For a moving target with non-zero range velocity component, it has two different range Doppler version, i.e., the introduced Doppler centroids are different in the same range bin of the two range Doppler patch. By using a feature value to enhance the Doppler spectrum, two peaks centered at the two Doppler centroids will appear, and thus the two fractional PRF parts are estimated. Based on the two centroid estimates, Doppler ambiguity is solved by the proposed DAS model. In addition, a stepped approximation-and-comparison algorithm is designed to compute a more accurate estimate of the range velocity component.

An azimuth velocity estimator (AVE) is designed based on the SDFP bank. It is more robust against noise and clutter theoretically than the traditional estimators that utilize such techniques as aufocusing and antenna pattern information.

The article is organized as follows. Range Doppler radar imaging algorithm is reviewed and azimuth smear length of the image of a point moving target is analyzed, and then a new GMTI scheme, including the MTD, DAS, SAC, and AVE, is designed and described in detail. Finally, the field and simulated data are used to confirm the effectiveness of the proposed scheme.

\section{Fundamentals \\ Radar imaging of a moving target}

The SAR uses the relative motion between the radar and the target to image the target. It can be airborne or spaceborne. It takes different modes in different applications, like stripmap SAR and spotlight SAR. Stripmap SAR includes broadside-looking SAR and squint SAR. This article treats airborne broadside-looking SAR. The addressed ideas and methods, however, may also apply to other mode of SAR. Typical algorithms for SAR imaging include the range-Doppler algorithm, the chirp-scaling algorithm, and the wavenumber-domain algorithm. In this article, we only consider the range-Doppler algorithm.

The analysis is performed in a typical slant-plane of a boresight strip-map SAR scenario shown in Figure 1. A moving target is located at $\left(r_{0}, y_{0}\right)$ with respect to the scene center when it is in the antenna bore-sight direction. It moves with a constant velocity $V_{\mathrm{a}}$ in azimuth and a constant velocity $V_{r}$ in range. The radar platform moves with a constant velocity $V_{\mathrm{p}}$. The bore-sight distance between the radar and the scene center is $R_{0}$. The time-frequency presentation of the transmitted waveform is shown at the top-right corner. The waveform consists of two kinds of pulses with the same chirp rate and pulse width $t_{\mathrm{p}}$, but modulated by two different carrier frequencies, i.e., $f_{1}$ and $f_{2}$ shown in the figure. The two carrier frequencies satisfy

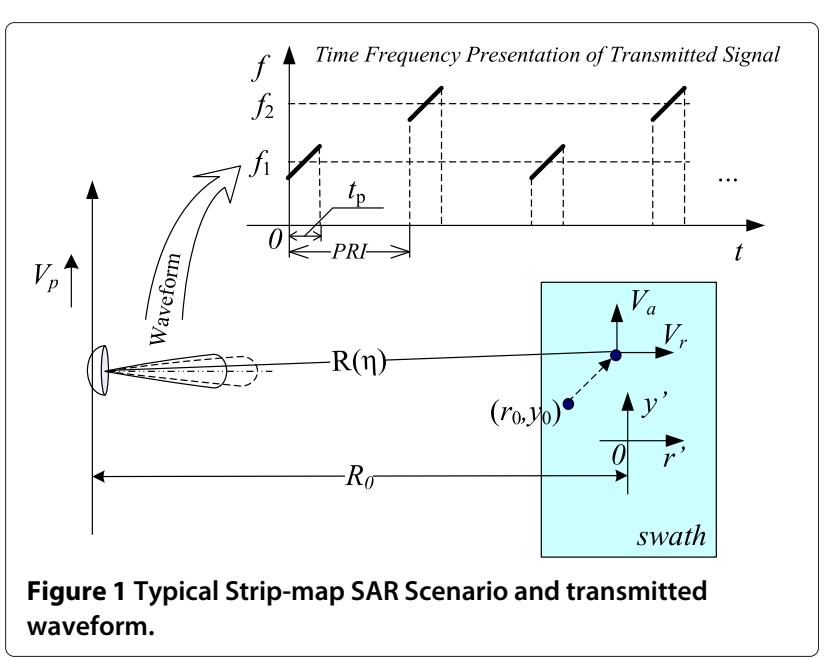


the relationship $B_{\mathrm{p}}<f_{2}-f_{1} \ll f_{1}$ and $f_{1} \gg B_{\mathrm{p}}$, where $B_{\mathrm{p}}$ is the bandwidth of transmitted signal. The two pulses are transmitted alternately by the same pulse repetition interval (PRI). For convenience, the two pulse bursts are denoted by $c_{1}$ and $c_{2}$, respectively.

The slow time is denoted by $\eta$, and when $\eta=0$, the scene center is in the antenna bore-sight direction. The distance between the platform and the moving target is a function of $\eta$ expressed by

$$
\begin{aligned}
R(\eta) & =\sqrt{\left(R_{0}+V_{\mathrm{r}} \eta\right)^{2}+\left[\left(V_{\mathrm{p}}-V_{\mathrm{a}}\right) \eta+y_{0}\right]^{2}} \\
& \approx R_{0}+V_{\mathrm{r}} \eta+\frac{\left[\left(V_{\mathrm{p}}-V_{\mathrm{a}}\right) \eta+y_{0}\right]^{2}}{2 R_{0}} .
\end{aligned}
$$

The demodulated return is

$$
s_{\mathrm{Rx}}(t, \eta)=e^{-j 2 \pi f_{\mathrm{c}} t_{0}} s_{\mathrm{Tx}}\left(t-t_{0}\right) \operatorname{rect}\left(\frac{t-t_{0}}{t_{\mathrm{p}}}\right),
$$

where $t_{0}=2 R(\eta) / c, c$ is the light speed, $t$ is the fast time, and the function $\operatorname{rect}(t)$ is defined by

$$
\operatorname{rect}(t)=\left\{\begin{array}{ll}
1, & |t| \leqslant 0.5 \\
0, & \text { otherwise }
\end{array} .\right.
$$

As we can see from (2) that the pulse compression in fast time domain does not influence the coefficient $e^{-j 2 \pi f_{c} t_{0}}$, the azimuth signal history.

Suppose that $\theta_{\mathrm{B}}$ is the antenna beam-width, the synthetic aperture length will be $L_{\mathrm{s}}=\theta_{\mathrm{B}} R_{0}$. According to kinetics theory, the synthetic aperture time is

$$
T_{\mathrm{s}}=\frac{\theta_{\mathrm{B}} R_{0}}{V_{\mathrm{p}}-V_{\mathrm{a}}}
$$

So ignoring the antenna beam-pattern and the constant term, the Doppler signal history of the moving target can be approximated by

$$
s_{\mathrm{m}}\left(\eta ; \boldsymbol{\xi}_{\mathrm{m}}\right)=\operatorname{rect}\left[\frac{\eta-\eta_{0}}{T_{\mathrm{s}}}\right] e^{-j \frac{4 \pi V_{\mathrm{r}}}{\lambda}\left(\eta-\eta_{0}\right)} e^{j \pi f_{\mathrm{dr}}^{m}\left(\eta-\eta_{0}\right)^{2}}
$$

where $\eta_{0}=y_{0} / V_{\mathrm{p}}, f_{\mathrm{dr}}^{m}=-2\left(V_{\mathrm{p}}-V_{\mathrm{a}}\right)^{2} /\left(\lambda R_{0}\right), \lambda$ is the carrier wavelength, and $\xi_{\mathrm{m}}=\left(r_{0}, y_{0}, V_{\mathrm{r}}, V_{\mathrm{a}}\right)$ is a vector describing the parameters of the moving target. Equation (4) indicates that the Doppler history of a moving target is a chirp signal centered at $f_{\mathrm{dc}}=-2 V_{\mathrm{r}} / \lambda$ having a Doppler rate $f_{\mathrm{dr}}^{\mathrm{m}}$. According to the matched filter theory [24], if the azimuth signal (4) is compressed by the filter

$$
H_{0}\left(f_{\mathrm{D}}\right)=e^{j \pi k_{\mathrm{s}} f_{\mathrm{D}}^{2}}
$$

the compressed result will be given by

$$
\begin{aligned}
s_{\mathrm{m}}^{\mathrm{c}}(\eta) & =\frac{\sin \left[\pi B_{\mathrm{D}}\left(\eta-\eta_{0}+\frac{V_{\mathrm{r}} R_{0}}{V_{\mathrm{P}}^{2}}\right)\right]}{\pi\left[\eta-\left(\eta_{0}-\frac{V_{\mathrm{r}} R_{0}}{V_{\mathrm{P}}^{2}}\right)\right]} * s_{\text {diff }}(\eta), \\
s_{\mathrm{diff}}(\eta) & =\mathcal{F}_{\substack{f_{\mathrm{D}} \rightarrow \eta \\
\left|f_{\mathrm{D}}\right| \leq B_{\mathrm{D}} / 2}}^{-1}\left[e^{-j \pi \alpha_{\mathrm{m}} k_{\mathrm{s}} f_{\mathrm{D}}^{2}}\right]
\end{aligned}
$$

for $\left|2 V_{\mathrm{r}} / \lambda\right|<f_{\mathrm{PRF}} / 4$. In (5), $k_{\mathrm{s}}=1 / f_{\mathrm{dr}}^{\mathrm{s}}$, and $f_{\mathrm{dr}}^{\mathrm{s}}=$ $-2 V_{\mathrm{p}}^{2} /\left(\lambda R_{0}\right)$. In (6), $\alpha_{\mathrm{m}}=k_{\mathrm{m}} / k_{\mathrm{s}}-1$ is referred to as the defocus coefficient, and the symbol " $*$ " means the convolution operation. It is observed that the range motion introduces a time-delay term resulting in the misplacement by $-V_{\mathrm{r}} R_{0} / V_{\mathrm{p}}$ relative to its real azimuth position. The time duration of (6) is

$$
\eta_{\mathrm{w}} \approx \frac{\lambda}{2 \theta_{\mathrm{B}} V_{\mathrm{p}}}+\left|\alpha_{\mathrm{m}}\right| \frac{R_{0} \theta_{\mathrm{B}}}{V_{\mathrm{p}}}
$$

and the corresponding azimuth smear length is

$$
\rho_{\text {smear }} \approx \rho_{\mathrm{a}}+\left|\alpha_{\mathrm{m}}\right| \frac{\lambda R_{0}}{2 \rho_{\mathrm{a}}},
$$

where $\rho_{\mathrm{a}}=\lambda /\left(2 \theta_{\mathrm{B}}\right)$ is the azimuth resolution [24] Equation (8) indicates that the image of the moving target is smeared approximately in $1+\left|\alpha_{\mathrm{m}}\right| \lambda R_{0} /\left(2 \rho_{\mathrm{a}}^{2}\right)$ azimuth resolution cells when its azimuth returns are compressed by (5). On the contrary, if the azimuth matched filter focuses the image of the moving target ideally, the image of background will be smeared by the same length as $\rho_{\text {smear }}$.

\section{Symmetric defocusing}

As the azimuth signal history takes the form

$$
s_{\mathrm{a}}(\eta)=e^{j \pi f_{\mathrm{dr}}^{\mathrm{m}} \eta^{2}},
$$

and according to the stationary phase theory [24], its Fourier transform is

$$
\mathrm{S}_{a}\left(f_{\mathrm{D}}, \alpha_{\mathrm{m}}\right)=e^{-j \pi k_{\mathrm{s}}\left(1+\alpha_{\mathrm{m}}\right) f_{\mathrm{D}}^{2}}
$$

if the azimuth signal, expressed by (9) or (10), is compressed by

$$
H\left(f_{\mathrm{D}}, \alpha\right)=e^{j \pi k_{\mathrm{s}}(1+\alpha) f_{\mathrm{D}}^{2}},
$$

then the image of the moving target will be smeared in

$$
M\left(\alpha, \alpha_{\mathrm{m}}\right)=1+\left|\alpha-\alpha_{\mathrm{m}}\right| \frac{\lambda R_{0}}{2 \rho_{\mathrm{a}}^{2}}
$$

azimuth resolution cells according to (8). Equation (12) tells that in a given SAR image the smeared length of a defocused moving target is proportional to $\left|\alpha-\alpha_{\mathrm{m}}\right|$. For a quadratic phase error, in the smeared image the energy tends to be spread uniformly over the distance of the smear for an unweighted aperture [11]. Without loss of generality, for a single point target with the intensity 
$|b|^{2}$ is located in the scene and defocused by (11), we will find that (1) if the target is stationary, then its image will be smeared in $M(\alpha, 0)$ resolution cells and the entire sharpness becomes $|b|^{4} / M(\alpha, 0)$, and that (2) if the target is a moving target characterized by $\boldsymbol{\xi}_{\mathrm{m}}$, its image will be smeared in $M\left(\alpha, \alpha_{\mathrm{m}}\right)$ resolution cells and the entire sharpness becomes $|b|^{4} / M\left(\alpha, \alpha_{\mathrm{m}}\right)$. The combination of $H\left(f_{\mathrm{D}}, \alpha\right)$ and $H\left(f_{\mathrm{D}},-\alpha\right)$ is defined as an SDFP. Inspired by the discussion above, an SDFP based MTD is described as follows.

First, the original SAR imagery is transformed to range Doppler domain and filtered by an SDFP, resulting in two defocused images. Second, the sharpness distribution images (SDI) of a defocused image are computed by

$$
S(i, j)=\sum_{p=(i-1) P / 2+1}^{(i+1) P / 2} \sum_{q=(j-1) Q / 2+1}^{(j+1) Q / 2}|I(p, q)|^{4},
$$

where $K$ is the pixels number in range and $L$ is that in azimuth of the SAR imagery, $P$ is the pixels in range and $Q$ is that in azimuth of a patch, $I(p, q)$ is the amplitude of the $(p, q)$-th pixel in the defocused image, $1 \leqslant i \leqslant M$, $1 \leqslant j \leqslant N, M=\lfloor 2 K / P\rfloor$, and $N=\lfloor 2 L / Q\rfloor$. Finally, the moving targets are indicated by comparing the two SDIs.

\section{Range velocity estimator}

\section{Baseband Doppler centroid indicator}

Figure 2 presents a sketch of the Doppler spectrum in the $r$-th range bin of the two patches containing the same moving target. The solid curve $A_{1}\left(f_{\mathrm{D}}, r\right)$ presents the Doppler spectrum of the patch from the original SAR imagery generated by $c_{1}$, and $A_{2}\left(f_{\mathrm{D}}, r\right)$ presents that of the patch from that by $c_{2}$. After $A\left(f_{\mathrm{D}}, r\right)$ is normalized by

$$
\bar{A}\left(f_{\mathrm{D}}, r\right)=\frac{B_{\mathrm{D}} A\left(f_{\mathrm{D}}, r\right)}{\int_{-B_{\mathrm{D}} / 2}^{B_{\mathrm{D}} / 2} A(f, r) d f},
$$

the normalized Doppler spectrum difference

$$
C_{\mathrm{r}}\left(f_{\mathrm{D}}\right)=\frac{\int_{f_{\mathrm{D}}-\Delta f / 2}^{f_{\mathrm{D}}+\Delta f / 2}\left|\bar{A}_{1}(f, r)-\bar{A}_{2}(f, r)\right| d f}{\int_{f_{\mathrm{D}}-\Delta f / 2}^{f_{\mathrm{D}}+\Delta f / 2}\left[\bar{A}_{1}(f, r)+\bar{A}_{2}(f, r)\right] d f}
$$

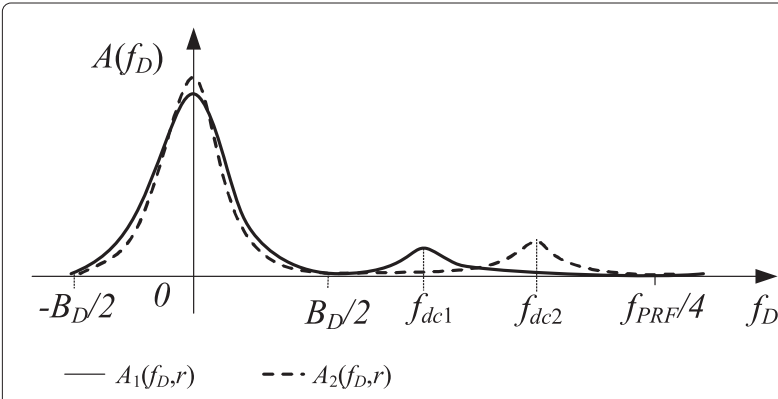

Figure 2 A sketch of Doppler amplitude spectra. is chosen as the baseband Doppler centroid indicator. In (15), $\Delta f=B_{\mathrm{D}} / N$, and $N$ is an arbitrary integer larger than 40 to our simulation experience.

It is apparent that $0 \leqslant C_{r}\left(f_{\mathrm{D}}\right) \leqslant 1$, and the criterion amplifies the weak Doppler spectrum of moving target and suppresses the strong Doppler spectrum of background. It is an important tool to analyze the Doppler centroid in this research. A typical criterion curve is shown in Figure 3. It can be seen that the two baseband Doppler centroid can be determined easily by this feature value.

\section{Doppler ambiguity solver}

The Doppler shifts of a moving target corresponding to the two carriers can be expressed by

$$
\begin{aligned}
& f_{\mathrm{d} 1}=f_{\mathrm{dc} 1}+m \cdot f_{\mathrm{PRF}} / 2=-\frac{2 V_{\mathrm{r}}}{\lambda_{1}}, \\
& f_{\mathrm{d} 2}=f_{\mathrm{d} c 2}+n \cdot f_{\mathrm{PRF}} / 2=-\frac{2 V_{\mathrm{r}}}{\lambda_{2}}
\end{aligned}
$$

where $m$ and $n$ are the Doppler ambiguity numbers, $\lambda_{1}$ is the wavelength of $c_{1}$, and $\lambda_{2}$ is that of $c_{2}$. So the Doppler ambiguity solver is modeled by

$$
\begin{aligned}
\hat{m}, \hat{n} t=\arg \min _{m, n} \mid \lambda_{1} f_{\mathrm{dc} 1} & -\lambda_{2} f_{\mathrm{dc} 2} \\
& +\frac{f_{\mathrm{PRF}}}{2}\left(\lambda_{1} m-\lambda_{2} n\right) \mid,
\end{aligned}
$$

s.t.

$$
\left\{\begin{array}{l}
m, n \in \mathbb{Z}, \lambda_{1}>\lambda_{2} \\
|m| \leqslant \frac{4 V_{\mathrm{R}}}{\lambda_{1} f_{\mathrm{PRF}}},|n| \leqslant \frac{4 V_{\mathrm{R}}}{\lambda_{2} f_{\mathrm{PRF}}}
\end{array}\right.
$$

with $V_{\mathrm{R}}$ being the possible maximum velocity in range. For example, on the freeway in China, $V_{\mathrm{R}}$ is no more than $35 \mathrm{~m} / \mathrm{s}$, while in urban, $V_{\mathrm{R}}$ will be set to $14 \mathrm{~m} / \mathrm{s}$.

If $\hat{m}$ and $\hat{n}$ are obtained from (18), then the Doppler shift can be estimated without ambiguity, and thus the range velocity can be estimated accurately.

Figure 4 presents the relationship between the baseband Doppler centroid and the range velocity for the two different carriers. The horizontal axis denotes the range velocity with $V_{1}=\lambda_{1} f_{\mathrm{PRF}} / 4$ and $V_{2}=\lambda_{2} f_{\mathrm{PRF}} / 4$, and the vertical axis denotes the baseband Doppler centroid. Line 1

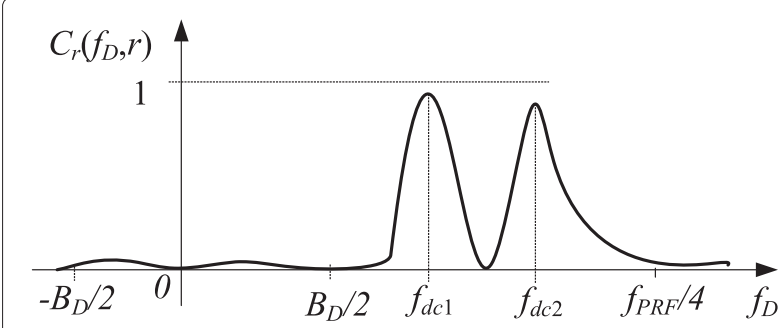

Figure 3 Normalized Doppler spectra difference. 


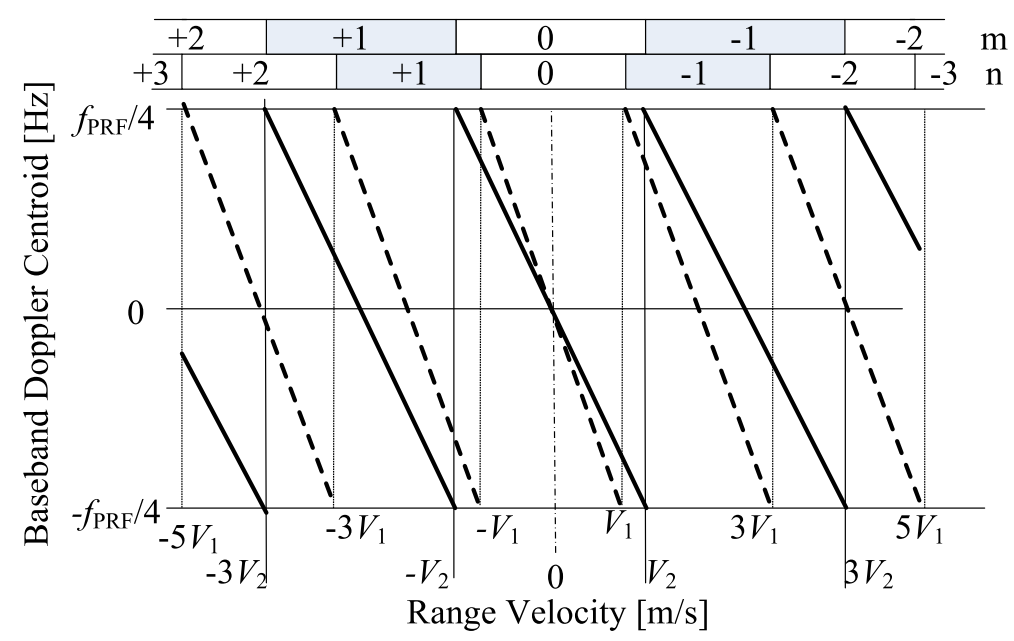

— line $1 \quad$ - - - line 2

Figure 4 Baseband Doppler centroid as a function of range velocity for the two carriers.

represents the baseband Doppler centroid as a function of the range velocity for $\mathrm{c}_{1}$, and line 2 represents that for $\mathrm{c}_{2}$. The integers in the upper text boxes are the ambiguity numbers, i.e., $m$ and $n$ in (16) and (17), in different range velocity intervals. For a given baseband Doppler centroid $f_{\mathrm{dc}}$, it corresponds to many different Doppler shift values. If both $f_{\mathrm{dc} 1}$ and $f_{\mathrm{dc} 2}$ are estimated, then $m$ and $n$ are determined accordingly.

Algorithm 1 presents pseudo-code for the function DAS which implements Doppler ambiguity solver associated with (18). It takes as input $f_{\mathrm{dc} 1}, f_{\mathrm{dc} 2}, f_{1}, f_{2}$, and $f_{\mathrm{PRF}}$, and returns $\hat{m}, \hat{n}$. It starts by calculating the maximum ambiguity number $M$ and $N$ with respect to $f_{1}$ and $f_{2}$ with possible maximum range velocity, then computes $f_{\mathrm{d} 1}$ and corresponding $f_{\mathrm{d} 2}^{0}$ using a probing ambiguity number $m$ from $-M$ to $M$ with an increment of 1 , therefore the Doppler shift difference, i.e., $\Delta f_{m, n}$, is obtained by $\left|f_{\mathrm{d} 2}-f_{\mathrm{d} 2}^{0}\right|$, where $f_{\mathrm{d} 2}$ is computed by using a probing ambiguity number $n$ from $-N$ to $N$ with an increment of 1 . Finally, the ambiguity number $\hat{m}$ and $\hat{n}$ are achieved and returned by finding the minimum value of $\Delta f_{m, n}$.

\section{Algorithm 1 Procedure for Doppler ambiguity solver}

Function $[\hat{m}, \hat{n}]:=\operatorname{DAS}\left(f_{\mathrm{dc} 1}, f_{\mathrm{dc} 2}, f_{1}, f_{2}, f_{\mathrm{PRF}}\right)$

Initialization: $V_{\mathrm{R}}:=$ possible maximum range velocity;

1: $\lambda_{1}:=\frac{c}{f_{1}}, \lambda_{2}:=\frac{c}{f_{2}}$, with $c$ being the light speed;

$$
\begin{aligned}
f_{\mathrm{d} 1}^{\mathrm{M}} & :=\frac{2 V_{\mathrm{R}}}{\lambda_{1}}, f_{\mathrm{d} 2}^{\mathrm{M}}:=\frac{2 V_{\mathrm{R}}}{\lambda_{2}} ; \\
M & :=\left\lceil\frac{2 f_{\mathrm{d} 1}^{\mathrm{M}}}{f_{\mathrm{PRF}}}\right\rceil, N:=\left\lceil\frac{2 f_{\mathrm{d} 2}^{\mathrm{M}}}{f_{\mathrm{PRF}}}\right\rceil ;
\end{aligned}
$$

2: for $m:=-\mathrm{M}$ to $\mathrm{M}$ (with an increment of 1 ) $f_{\mathrm{d} 1}:=f_{\mathrm{dc} 1}+\mathrm{m} \cdot f_{\mathrm{PRF}} / 2 ; V_{\mathrm{r}}:=-\lambda_{1} f_{\mathrm{d} 1} / 2 ; f_{\mathrm{d} 2}^{0}:=-\frac{2 V_{\mathrm{r}}}{\lambda_{2}}$;

3: for $\mathrm{n}:=-\mathrm{N}$ to $\mathrm{N}$ (with an increment of 1 ) end for $[m]$;

$$
\begin{aligned}
& f_{\mathrm{d} 2}:=f_{\mathrm{dc} 2}+\mathrm{n} \cdot f_{\mathrm{PRF}} / 2 ; \\
& \Delta f_{m, n}=\left|f_{\mathrm{d} 2}^{0}-f_{\mathrm{d} 2}\right| ;
\end{aligned}
$$

end for $[n]$;

4: Find the minimum value from $\left\{\Delta f_{m, n}\right\}$, and get the corresponding index $\hat{m}$ and $\hat{n}$;

\section{Stepped approximation-and-comparison algorithm}

From Figure 4, we see that a target which moves at low speed in range direction may result in the superposition between the Doppler spectrum of background and that of the moving target. It will lead to the wrong determination of Doppler spectrum peak locations. On the other hand, the estimate of $f_{\mathrm{dc} 1}$ and $f_{\mathrm{dc} 2}$ may become inaccurate because of noise, platform turbulence, and clutter etc. An algorithm named SAC is developed to get a finer baseband Doppler centroid.

If the coarse baseband Doppler centroid values are $f_{\mathrm{dc} 1}$ and $f_{\mathrm{dc} 2}$, and the Doppler ambiguity numbers $\hat{m}$ and $\hat{n}$ are obtained from (18), then the unambiguous Doppler shifts can be described by

$$
\begin{aligned}
& f_{\mathrm{d} 1}=f_{\mathrm{dc} 1}+\Delta f_{\mathrm{d}}+\hat{m} \frac{f_{\mathrm{PRF}}}{2}, \\
& f_{\mathrm{d} 2}=f_{\mathrm{dc} 2}-\Delta f_{\mathrm{d}}+\hat{n} \frac{f_{\mathrm{PRF}}}{2},
\end{aligned}
$$

where $\Delta f_{\mathrm{d}}$ is a modified Doppler shift value derived from

$$
\begin{gathered}
\Delta \hat{f}_{\mathrm{d}}=\arg \min _{\Delta f_{\mathrm{d}}} \mid \lambda_{1} f_{\mathrm{dc} 1}-\lambda_{2} f_{\mathrm{dc} 2}+\frac{f_{\mathrm{PRF}}}{2}\left(\lambda_{1} \hat{m}-\lambda_{2} \hat{n}\right) \\
+\left(\lambda_{1}+\lambda_{2}\right) \Delta f_{\mathrm{d}} \mid
\end{gathered}
$$


s.t.

$$
\left|\Delta f_{\mathrm{d}}\right| \leqslant \frac{B_{\mathrm{D}}}{2}
$$

Algorithm 1 presents the computation procedure of accurate $f_{\mathrm{dc} 1}$ and $f_{\mathrm{dc} 2}$. It takes as input $f_{\mathrm{dc} 1}, f_{\mathrm{dc} 2}, \hat{m}, \hat{n}$, $f_{\mathrm{PRF}}, f_{1}$, and $f_{2}$, where $\hat{m}$ and $\hat{n}$ are the ambiguity numbers computed by DAS, and returns two finer unambiguous Doppler shifts of the moving target. It starts by defining an approximation step $\Delta f=B_{\mathrm{D}} /(2 K)$, where $K$ is an arbitrary integer (here we assume that $K$ is equal to 100), and computes the initial unambiguous shifts $f_{\mathrm{d} 1}^{0}$ and $f_{\mathrm{d} 2}^{0}$, respectively. Then for a given $k$ from $-K$ to $K$ with increment of 1 , the new $f_{\mathrm{d} 1}$ and $f_{\mathrm{d} 2}$ are updated using (19) and (20) with the substitution of $k \Delta f$ to $\Delta f_{\mathrm{d}}$, and thus two corresponding range velocities resulting from the updated unambiguous Doppler shift are achieved and the difference between the two velocities is stored in the sequence $\left\{\Delta V_{k}\right\}$. Finally, by searching the minimum value of $\left\{\Delta V_{k}\right\}$, the finer unambiguous Doppler shifts are obtained and returned.

\section{Algorithm 2 Procedure for stepped}

\section{approximation-and-comparison algorithm}

Function $\left[\hat{f}_{\mathrm{dc} 1}, \hat{f}_{\mathrm{dc} 2}\right]:=\operatorname{SAC}\left(f_{\mathrm{dc} 1}, f_{\mathrm{dc} 2}, \hat{m}, \hat{n}, f_{\mathrm{PRF}}, f_{1}, f_{2}\right)$

Initialization: $K:=$ An integer larger than 100;

1: $\quad \lambda_{1}=\frac{c}{f_{1}}, \lambda_{2}=\frac{c}{f_{2}}$

$f_{\mathrm{d} 1}^{0}:=\hat{m} \cdot f_{\mathrm{PRF}} / 2+f_{\mathrm{dc} 1}, f_{\mathrm{d} 2}^{0}:=\hat{n} \cdot f_{\mathrm{PRF}} / 2+f_{\mathrm{dc} 2}, \Delta f:=\frac{B_{\mathrm{D}}}{2 K} ;$

2: for $k:=-K$ to $K$ (with an increment of 1 )

$f_{\mathrm{d} 1}:=f_{\mathrm{d} 1}^{0}+k \cdot \Delta f, f_{\mathrm{d} 2}:=f_{\mathrm{d} 2}^{0}-k \cdot \Delta f$;

$V_{\mathrm{r} 1}:=-\frac{\lambda_{1} f_{\mathrm{d} 1}}{2}, V_{\mathrm{r} 2}:=-\frac{\lambda_{2} f_{\mathrm{d} 2}}{2}$;

$\Delta V_{k}:=\left|V_{\mathrm{r} 1}-V_{\mathrm{r} 2}\right|$;

end for $[k]$

3: Find the minimum value from $\left\{\Delta V_{k}\right\}$, and get the modified index $k$;

4: $\hat{f}_{\mathrm{dc} 1}:=f_{\mathrm{d} 1}^{0}+k \Delta f, \hat{f}_{\mathrm{dc} 2}:=f_{\mathrm{d} 2}^{0}-k \Delta f$;

When the finer Doppler shift $\hat{f}_{\mathrm{d} 1}$ and $\hat{f}_{\mathrm{d} 2}$ are achieved, two estimates of the range velocity are computed from

$$
\begin{aligned}
& \hat{V}_{\mathrm{r} 1}=-\frac{\lambda_{1} \hat{f}_{\mathrm{d} 1}}{2}, \\
& \hat{V}_{\mathrm{r} 2}=-\frac{\lambda_{2} \hat{f}_{\mathrm{d} 2}}{2} .
\end{aligned}
$$

Finally the average range velocity component is

$$
\hat{V}_{\mathrm{r}}=\frac{\hat{V}_{\mathrm{r} 1}+\hat{V}_{\mathrm{r} 2}}{2} \text {. }
$$

\section{Further discussion}

The primary condition for DAS and SAC is that the Doppler bandwidth is far smaller than $f_{\mathrm{PRF}}$ (at least no more than $\left.f_{\mathrm{PRF}} / 4\right)$. Under this condition, if $f_{\mathrm{d} 1}$ and $f_{\mathrm{d} 2}$ are confined by

$$
\begin{aligned}
& k_{1} \frac{f_{\mathrm{PRF}}}{2}-\frac{B_{\mathrm{D}}}{2}<f_{\mathrm{d} 1}<k_{1} \frac{f_{\mathrm{PRF}}}{2}+\frac{B_{\mathrm{D}}}{2}, \\
& k_{2} \frac{f_{\mathrm{PRF}}}{2}-\frac{B_{\mathrm{D}}}{2}<f_{\mathrm{d} 2}<k_{2} \frac{f_{\mathrm{PRF}}}{2}+\frac{B_{\mathrm{D}}}{2},
\end{aligned}
$$

where $k_{1}$ and $k_{2}$ are integers, two cases will appear in general:

\section{Both $k_{1}$ and $k_{2}$ are equal to zero}

In this case, the peaks will disappear in $C_{\mathrm{r}}\left(f_{\mathrm{d}}\right)$, so the range velocity component cannot be measured, thus the minimum measurable range velocity is

$$
\left|V_{\min , \mathrm{r}}\right|=\lambda_{2} \frac{B_{\mathrm{D}}}{4}, \text { for } \lambda_{1}>\lambda_{2}
$$

\section{Either of $k_{1}$ and $k_{2}$ is not equal to zero}

If $k_{1} \neq 0$, and $f_{\mathrm{d} 1}$ satisfies $(25)$, then $f_{\mathrm{d} 1}$ is

$$
f_{\mathrm{d} 1}=k_{1} \frac{f_{\mathrm{PRF}}}{2}+\delta f_{\mathrm{d}}
$$

where $\left|\delta f_{\mathrm{d}}\right|<B_{\mathrm{D}} / 2 . f_{\mathrm{d} 2}$ can be expressed by

$$
f_{\mathrm{d} 2}=\frac{\lambda_{1}}{\lambda_{2}} k_{1} \frac{f_{\mathrm{PRF}}}{2}+\frac{\lambda_{1}}{\lambda_{2}} \delta f_{\mathrm{d}}
$$

If $f_{\mathrm{d} 1}$ and $f_{\mathrm{d} 2}$ are wrapped by the same ambiguity number, then $\lambda_{1}$ and $\lambda_{2}$ have the relationship

$$
\frac{\lambda_{1}}{\lambda_{2}} \leqslant \frac{M+1}{M}
$$

where $M$ is the maximum ambiguity number describe in Algorithm 1 . To make $f_{\mathrm{dc} 2}$ detectable, the following requirement should be satisfied

$$
\left(\frac{\lambda_{1}}{\lambda_{2}}-1\right) \frac{f_{\mathrm{PRF}}}{2}+\frac{\lambda_{1}}{\lambda_{2}} \delta f_{\mathrm{d}} \geqslant \frac{B_{\mathrm{D}}}{2} .
$$

So $\lambda_{1}$ and $\lambda_{2}$ satisfy

$$
\frac{\lambda_{1}}{\lambda_{2}} \geqslant 1+\frac{B_{\mathrm{D}}}{f_{\mathrm{PRF}}} \text {. }
$$

Equation (32) tells us that when the dual-frequency SAR system parameters, such as carrier frequencies, PRF, radar velocity and azimuth resolution, satisfy both (30) and (32), the range velocity component of a moving target can be computed without unambiguity.

If $f_{\mathrm{dc} 2}$ is determined, then the ambiguity numbers $\hat{m}$ and $\hat{n}$ can be computed from

$$
[\hat{m}, \hat{n}]=\arg \min _{m, n}\left|\lambda_{2} f_{\mathrm{dc} 2}+\left(\lambda_{2} n-\lambda_{1} m\right) \frac{f_{\mathrm{PRF}}}{2}\right| .
$$

In a similar way, when $k_{2} \neq 0$ and $f_{\mathrm{d} 2}$ satisfy (26), the ambiguity numbers $\hat{m}$ and $\hat{n}$ can be deduced from

$$
[\hat{m}, \hat{n}]=\arg \min _{m, n}\left|\lambda_{1} f_{\mathrm{dc} 1}+\left(\lambda_{1} m-\lambda_{2} n\right) \frac{f_{\mathrm{PRF}}}{2}\right| .
$$




\section{Azimuth displacement correction}

It is noteworthy that (6) is established based on the condition that $\left|V_{\mathrm{r}} / \lambda\right|<f_{\mathrm{PRF}} / 4$, and the moving target will be displaced by $-V_{\mathrm{r}} R_{0} / V_{\mathrm{p}}$ in azimuth direction. It seems that the azimuth displacement is independent of the carrier frequency. However, when the target is moving so fast that $\left|V_{\mathrm{r}} / \lambda\right|>f_{\mathrm{PRF}} / 4$, the displacement equation must be modified.

Ignoring the azimuth velocity component, the baseband Doppler history will be

$$
\phi(\eta)=-\frac{4 \pi}{\lambda} R_{0}-2 \pi\left(\frac{2 V_{\mathrm{r}}}{\lambda}+n \frac{f_{\mathrm{PRF}}}{2}\right) \eta-2 \pi \frac{V_{\mathrm{P}}^{2}}{\lambda R_{0}} \eta^{2},
$$

where $n$ is the ambiguity number of the Doppler shift $f_{\mathrm{d}}=$ $-2 V_{\mathrm{r}} / \lambda$. The azimuth displacement will be given by

$$
\Delta a=-\frac{4 V_{\mathrm{r}}+n \lambda f_{\mathrm{PRF}}}{4 V_{\mathrm{p}}} R_{0} .
$$

We see that if the Doppler ambiguity appears, the azimuth displacement of a moving target must be modified by (36). This equation is an extension to $[25,26]$ and is the basic model for correcting the target's real azimuth position in a SAR image.

\section{Azimuth velocity estimator \\ Theoretical analysis}

When a patch containing a moving target is defocused by $H\left(f_{\mathrm{D}}, \alpha\right)$ and $H\left(f_{\mathrm{D}},-\alpha\right)$, two derivative SAR image patches, denoted by $P_{1}$ and $P_{2}$, are achieved simultaneously. Let $\Delta V_{\mathrm{a}}$ be an arbitrary probing azimuth velocity, and in general both $\Delta V_{\mathrm{a}}$ and $V_{\mathrm{a}}$ are far less than $V_{\mathrm{p}}$, which means that $\alpha \approx 2 \Delta V_{\mathrm{a}} / V_{\mathrm{p}}$. Equation (12) can be rewritten by

$$
M\left(\Delta V_{\mathrm{a}}, V_{\mathrm{a}}\right)=1+\frac{\left|V_{\mathrm{a}}-\Delta V_{\mathrm{a}}\right|}{V_{\mathrm{p}}} \frac{\lambda R_{0}}{\rho_{\mathrm{a}}^{2}} .
$$

For simplicity, we assume that the patch contains a moving point target and a stationary point target, and the two targets have the intensity $|b|^{2}$ and $|g|^{2}$, respectively. The patch $\mathrm{P}_{1}$ has the sharpness

$$
S_{\mathrm{p} 1}=\frac{|b|^{4}}{M\left(\Delta V_{\mathrm{a}}, V_{\mathrm{a}}\right)}+\frac{|g|^{4}}{M\left(\Delta V_{\mathrm{a}}, 0\right)},
$$

and $\mathrm{P}_{2}$ has the sharpness

$$
S_{\mathrm{p} 2}=\frac{|b|^{4}}{M\left(-\Delta V_{\mathrm{a}}, V_{\mathrm{a}}\right)}+\frac{|g|^{4}}{M\left(\Delta V_{\mathrm{a}}, 0\right)} .
$$

The sharpness difference between $S_{\mathrm{p} 1}$ and $S_{\mathrm{p} 2}$

$$
f\left(\Delta V_{\mathrm{a}}\right)=\frac{|b|^{4}}{M\left(\Delta V_{\mathrm{a}}, V_{\mathrm{a}}\right)}-\frac{|b|^{4}}{M\left(-\Delta V_{\mathrm{a}}, V_{\mathrm{a}}\right)}
$$

will be used as a feature to estimate the azimuth velocity component. The feature value is robust because it tries to alleviate the influence of clutters and interferences.

Let's discuss (40). Allowing for the symmetry of the SDFP, the probing velocity $\Delta V_{\mathrm{a}}$ is set to be larger than zero. For $\left.V_{\mathrm{a}}>0,1\right)$ in the case of $0<\Delta V_{\mathrm{a}} \leqslant V_{\mathrm{a}}$, the differential of $f\left(\Delta V_{\mathrm{a}}\right)$ is

$$
\frac{\mathrm{d} f\left(\Delta V_{\mathrm{a}}\right)}{\mathrm{d} \Delta V_{\mathrm{a}}}=\frac{2 \lambda R_{0} V_{p} \rho_{a}^{2}|b|^{4}\left[\left(V_{p} \rho_{a}^{2}+\lambda R_{0} V_{a}\right)^{2}+\left(\lambda R_{0} \Delta V_{a}\right)^{2}\right]}{\left[\left(V_{\mathrm{p}} \rho_{\mathrm{a}}^{2}+\lambda R_{0} V_{\mathrm{a}}\right)^{2}-\left(\lambda R_{0} \Delta V_{\mathrm{a}}\right)^{2}\right]^{2}}>0 .
$$

And 2) in the case of $\Delta V_{\mathrm{a}}>V_{\mathrm{a}}$, the differential of $f\left(\Delta V_{\mathrm{a}}\right)$ is

$$
\frac{\mathrm{d} f\left(\Delta V_{\mathrm{a}}\right)}{\mathrm{d} \Delta V_{\mathrm{a}}}=-\frac{4 \lambda^{2} R_{0}^{2} V_{a} V_{p} \rho_{a}^{2}|b|^{4}\left(V_{p} \rho_{a}^{2}+\lambda R_{0} \Delta V_{a}\right)}{\left[\left(V_{p} \rho_{a}^{2}+\lambda R_{0} \Delta V_{a}\right)^{2}-\left(\lambda R_{0} V_{a}\right)^{2}\right]^{2}}<0 .
$$

The two cases show that (1) when $0<\Delta V_{\mathrm{a}} \leqslant V_{\mathrm{a}}$, the sharpness difference is a monotonic increasing function and it reaches the maximum value at the point where $\Delta V_{\mathrm{a}}=V_{\mathrm{a}}$, and (2) when $\Delta V_{\mathrm{a}}>V_{\mathrm{a}}$, the sharpness difference is a monotonic decreasing function and it reaches the maximum value at the point where $\Delta V_{\mathrm{a}}=V_{\mathrm{a}}$. In addition, it infinitely approaches zero with the increment of $\Delta V_{\mathrm{a}}$.

In the case of $V_{\mathrm{a}}<0$, the following conclusions can be drawn: (1) when $0<\Delta V_{\mathrm{a}} \leqslant-V_{\mathrm{a}}$, the sharpness difference is a monotonic decreasing function and it reaches the minimum value at the point where $\Delta V_{\mathrm{a}}=-V_{\mathrm{a}}$, and (2) when $\Delta V_{\mathrm{a}}>-V_{\mathrm{a}}$, the sharpness difference is a monotonic increasing function and it reaches the minimum value at the point where $\Delta V_{\mathrm{a}}=V_{\mathrm{a}}$. In addition, it infinitely approaches zero with the decrement of $\Delta V_{\mathrm{a}}$.

Figure 5 presents a sketch of $f\left(\Delta V_{\mathrm{a}}\right)$ for two cases: $V_{\mathrm{a} 1}>0$ (for target 1) and $V_{\mathrm{a} 2}<0$ (for target 2). We see that the maximum sharpness difference is located at the point where $\Delta V_{\mathrm{a}}=\left|V_{\mathrm{a}}\right|$. If the maximum sharpness difference is less than zero, then the corresponding target is moving in the opposite direction of the platform. If the maximum sharpness difference is larger than zero, then the target is moving in the direction of the platform.

\section{Implementation}

From the discussions above, the azimuth velocity estimator can be modeled by

$$
\hat{V}_{\mathrm{a}}=\arg \max _{\Delta V_{\mathrm{a}}} f\left(\Delta V_{\mathrm{a}}\right) .
$$

Figure 6 presents a flowchart of AVE. A complex-valued patch containing a detected moving target is transformed to range Doppler domain by FFTs. The probing azimuth velocity $\Delta V$ is set from $V_{\mathrm{a}}^{0}$ to $V_{\mathrm{a}}^{\mathrm{d}}$ with increment of $\delta V_{\mathrm{a}}$. For each $\Delta V_{\mathrm{a}}$, the coefficient $\alpha$ is computed and an SDFP 


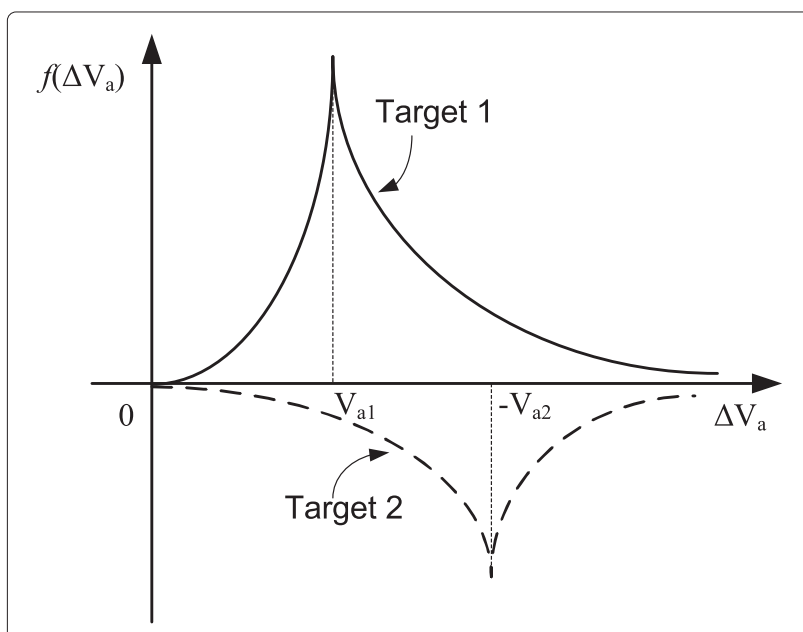

Figure $5 \mathrm{~A}$ sketch of sharpness difference as functions of $\Delta V_{\mathrm{a}}$. is established to defocus the range Doppler map resulting in two defocused images, and the sharpness difference between the two images is sent to an array named $s_{\mathrm{d}}$. The azimuth velocity estimate will appear at the position where $s_{\mathrm{d}}$ reaches the peak.

Two patches containing the same target are isolated from the two original SAR images and processed by the AVE. If the two estimates of the azimuth velocity component are denoted by $\hat{V}_{\mathrm{a} 1}$ and $\hat{V}_{\mathrm{a} 2}$, then the final azimuth velocity value is synthesized by

$$
\hat{V}_{\mathrm{a}}=\frac{\hat{V}_{\mathrm{a} 1}+\hat{V}_{\mathrm{a} 2}}{2}
$$

\section{Discussion}

Let us discuss the minimum detectable azimuth velocity component $V_{\text {min,a. }}$. It is relative to the threshold value $f_{\text {th }}$ which is used to identify the moving target area. We select

\section{A patch containing a} moving target

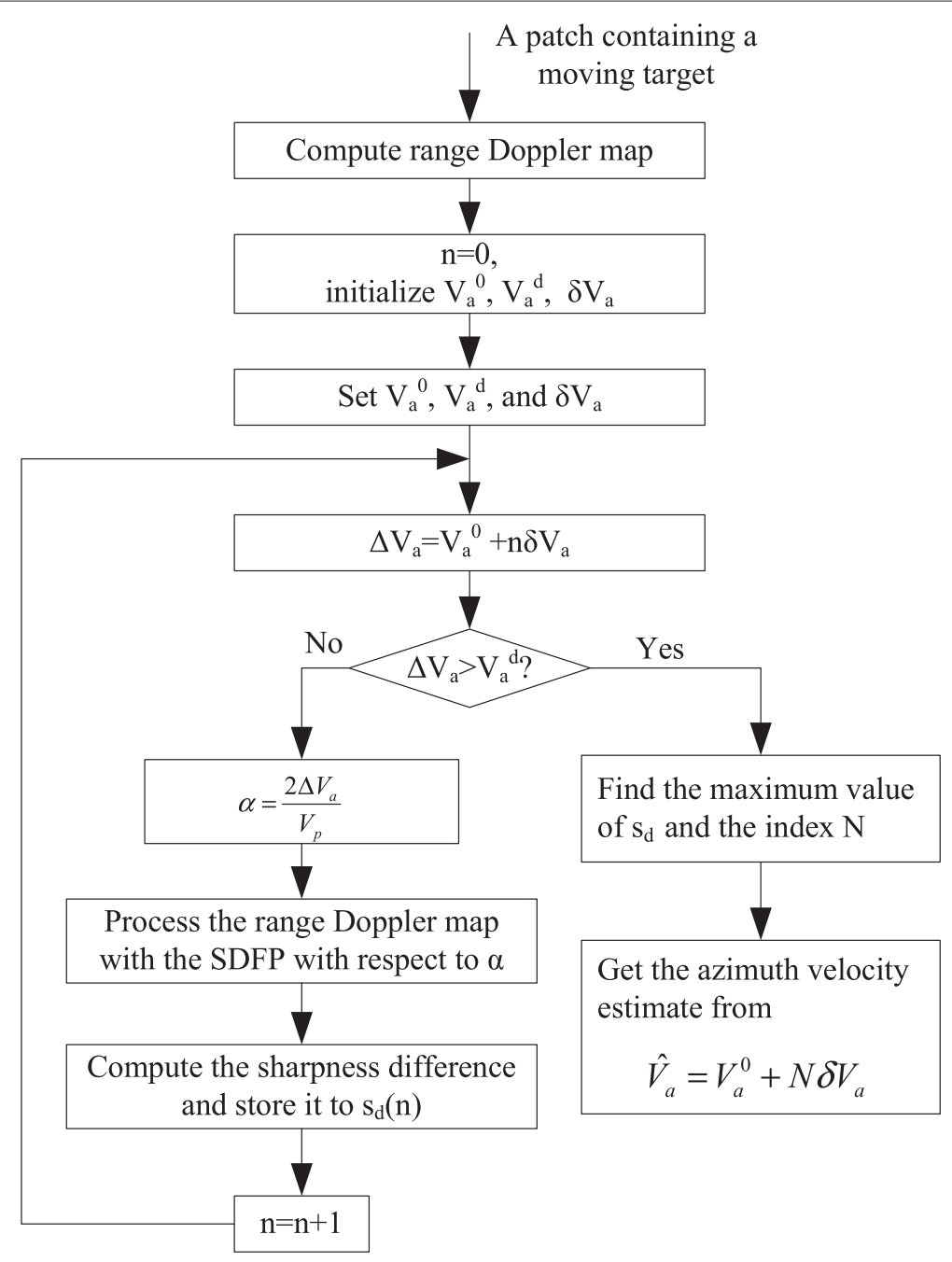

Figure 6 Flowchart of AVE. 


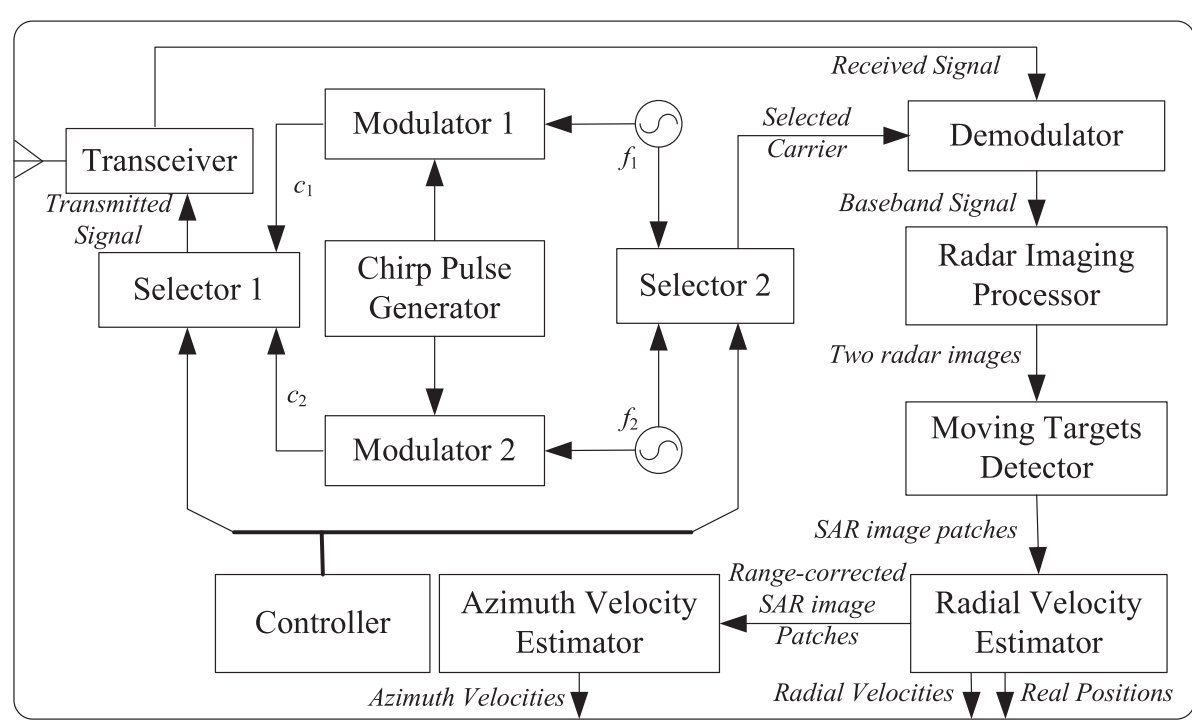

Figure 7 GMTI scheme using a dual-frequency SAR.

this value according to false-alarm probabilities and detection probability specification. According to our experience and simulation results, the feature value $f(i, j)$ follows a half positive Gaussian distribution approximately

$$
p(f)=\frac{2}{\sqrt{2 \pi} \sigma} e^{-\frac{f^{2}}{2 \sigma^{2}}}, f \geqslant 0
$$

So, from

$$
P_{\mathrm{F}}=\frac{2}{\sqrt{2 \pi} \sigma} \int_{f_{\mathrm{th}}}^{\infty} e^{-\frac{f^{2}}{2 \sigma^{2}}} d f=\operatorname{erfc}\left(\frac{f_{\mathrm{th}}}{\sqrt{2} \sigma}\right)
$$

we see that if a constant false alarm ratio $P_{\mathrm{F}}$ is given, the threshold value $f_{\text {th }}$ can be determined.

We estimate the unknown parameter $\sigma$ of (45) using MATLAB's fminsearch routine, which uses the NelderMead parameter search procedure [27,28]. As $V_{\text {min,a }} \ll$ $V_{\mathrm{p}}$, we have $\alpha_{\min } \approx 2 V_{\min , \mathrm{a}} / V_{\mathrm{p}}$. Let $f_{\max }$ denote the sharpness of the ideally focused target, and the sharpness of a moving target with azimuth velocity $V_{\text {min,a }}$ will be

$$
f_{\min , \mathrm{a}}=\frac{f_{\max }}{1+\frac{\alpha_{\mathrm{m}} \lambda R_{0}}{\rho_{\mathrm{a}}^{2}}}
$$

in the SAR image having focused background. As $f_{\min , \mathrm{a}} \geqslant$ $f_{\text {th }}, \alpha_{\text {min }}$ approximately satisfies

$$
1-\frac{1}{1+\frac{\alpha_{\min \lambda R_{0}}}{\rho_{a}^{2}}} \geqslant \frac{f_{\mathrm{th}}}{f_{\max }} .
$$

As a result, the minimum detectable azimuth velocity is approximately

$$
V_{\mathrm{min}, \mathrm{a}} \approx \frac{\epsilon \rho_{a}^{2} V_{\mathrm{p}}}{2(1-\epsilon) \lambda R_{0}}
$$

where $\epsilon=f_{\text {th }} / f_{\max }$.

\section{Experiments}

\section{Framework}

Based on the discussions above, a sketch of the proposed single antenna SAR using a dual frequency chirp waveform and the corresponding GMTI procedure is shown in Figure 7. In this figure, the controller controls the selector 1 to pass the two kinds of pulses to the transceiver alternately, and controls the selector 2 to pass the carrier being used to the demodulators to get the corresponding baseband returns. The radar imaging processor processes the returns and generates two original SAR images. The bottom-right of the figure shows the GMTI flowchart. First, the two original SAR images are processed by the MTD, and as a result, image patches that contain the detected moving targets are achieved. Second, the isolated patches are transmitted to the RVE to estimate their range velocity components and their real azimuth positions. Finally, the range-velocity-compensated patches are sent to the AVE to estimate their azimuth velocity components.

Table 1 System parameters of field data and simulation

\begin{tabular}{clll}
\hline Symbol & Description & \multicolumn{2}{c}{ Value } \\
\cline { 3 - 4 } & & Field data & Simulation \\
\hline$R_{0}$ & Range to scene center & $40800 \mathrm{~m}$ & $10000 \mathrm{~m}$ \\
$V_{\mathrm{p}}$ & Platform velocity & $218 \mathrm{~m} / \mathrm{s}$ & $200 \mathrm{~m} / \mathrm{s}$ \\
$T_{\mathrm{p}}$ & Pulse width & $20 \mu \mathrm{s}$ & $10 \mu \mathrm{s}$ \\
$f_{\mathrm{p}}$ & PRF & $1200 \mathrm{~Hz}$ & $4000 \mathrm{~Hz}$ \\
$f_{\mathrm{c}}$ & Carrier frequency & $9.6 \mathrm{GHz}$ & $10 \mathrm{GHz}, 12 \mathrm{GHz}$ \\
$B_{\mathrm{w}}$ & Signal bandwidth & $400 \mathrm{MHz}$ & $200 \mathrm{MHz}$ \\
$\rho_{\mathrm{r}}$ & Range resolution & $0.5 \mathrm{~m}$ & $1.0 \mathrm{~m}$ \\
$\rho_{\mathrm{a}}$ & Azimuth resolution & $0.5 \mathrm{~m}$ & $1.0 \mathrm{~m}$ \\
\hline
\end{tabular}



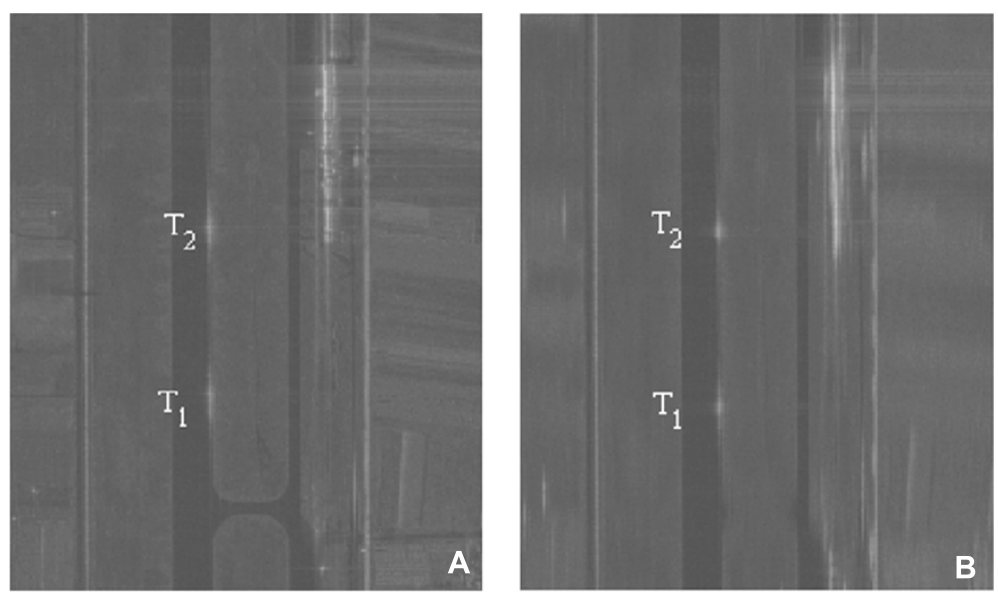

Figure 8 A field SAR image containing two moving vehicles (A) and its refocused image (B).

As a result, the range and azimuth velocity components together with their real azimuth positions are worked out.

We use a patch of real complex-valued SAR image to verify the MTD and AVE algorithms. The image is achieved by using one carrier, so it is cannot be used to determine the range velocity component of a moving target. In order to evaluate the performance of the RVE, with no field data available so far, computer simulations are conducted to confirm the effectiveness of the proposed algorithms. The raw data are simulated by using the model proposed in [29]. The model can simulate the effects of moving targets, and it is both analytically and quantitatively validated. We think that the following simulation based on this model can validate our idea.

\section{AVE verification with field data}

The complex-valued SAR image is collected near an airport in Shaanxi, China. The radar system and geometry parameters are shown in Table 1. In the illuminated scene, the trajectory of the SAR is parallel to the runway, and two vehicles are running at the speed of about $5.0 \mathrm{~m} / \mathrm{s}$ along the runway successively.

We isolated a patch of size 6528 pixels in range by 5120 pixels in azimuth near the moving targets from the given image. The patch is shown in Figure 8A. In this figure, the scene is about $765 \mathrm{~m}$ in azimuth and $600 \mathrm{~m}$ in range. The two known vehicles are labeled by $\mathrm{T}_{1}$ and $\mathrm{T}_{2}$. We see that the background is focused fairly well while the image of the two targets are smeared.

We defocused the patch by using an SDFP with $\alpha=$ 0.0985 (correspondingly, $\Delta V_{a}=10 \mathrm{~m} / \mathrm{s}$ ). The moving targets are detected correctly. An SDFP bank is constructed to estimate the azimuth velocity components of the two vehicles. In the experiment, $\Delta V_{a}$ ranges from $0 \mathrm{~m} / \mathrm{s}$ to $30 \mathrm{~m} / \mathrm{s}$ with an increment of $0.1 \mathrm{~m} / \mathrm{s}$. The range velocity components are assumed to be $0 \mathrm{~m} / \mathrm{s}$. Figure 9 presents the normalized feature curves for $T_{1}$ and $T_{2}$. From the figure, the azimuth velocity estimates are $-4.8 \mathrm{~m} / \mathrm{s}$ and $-5.0 \mathrm{~m} / \mathrm{s}$, respectively.

We refocused the moving target based on the two azimuth velocity component estimates. The result is shown in Figure 8B. It shows that the two vehicles are focused well while the background is smeared.

\section{Performance evaluation by computer simulation}

In the simulation, the background is a patch of terrain selected near the Kun-yu mountain in Shandong, China. It is about $200 \mathrm{~m}$ in azimuth and $160 \mathrm{~m}$ in range. The most important properties of the dual-frequency SAR, the moving targets and the background are incorporated. The radar system and geometry parameters are presented in Table 1.

The parameters of six moving targets labeled by $\mathrm{T}_{1} \sim$ $\mathrm{T}_{6}$ are shown in the columns $P^{\dagger}, V_{\mathrm{a}}$, and $V_{\mathrm{r}}$ of Table 2 .

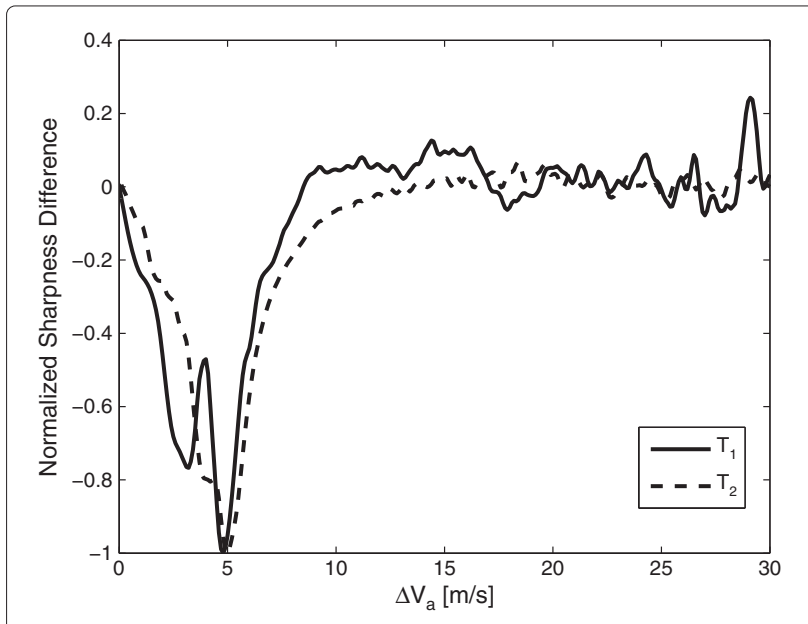

Figure 9 Feature curves of $T_{1}$ and $T_{2}$ in the field SAR image. 
Table 2 Moving target parameters and their estimated results

\begin{tabular}{|c|c|c|c|c|c|c|c|c|c|c|c|c|}
\hline Target & $\begin{array}{l}\mathbf{P}^{\dagger} \\
{[\mathrm{m}]}\end{array}$ & $\begin{array}{c}V_{a} \\
{[\mathrm{~m} / \mathrm{s}]}\end{array}$ & $\begin{array}{c}\mathrm{V}_{\mathrm{r}} \\
{[\mathrm{m} / \mathrm{s}]}\end{array}$ & $\begin{array}{l}\mathbf{f}_{\mathrm{dc} 1} \\
{[\mathrm{~Hz}]}\end{array}$ & $\begin{array}{l}f_{\mathrm{dc} 2} \\
{[\mathrm{~Hz}]}\end{array}$ & $\hat{\mathbf{m}}$ & $\hat{\mathbf{n}}$ & $\begin{array}{c}\hat{\mathrm{V}}_{\mathrm{r}} \\
{[\mathrm{m} / \mathrm{s}]}\end{array}$ & $\begin{array}{c}\hat{\mathrm{V}}_{\mathrm{a}} \\
{[\mathrm{m} / \mathrm{s}]}\end{array}$ & $\begin{array}{l}\hat{P}_{1}^{\dagger} \\
{[\mathrm{m}]}\end{array}$ & $\begin{array}{l}\hat{P}_{2}^{\dagger} \\
{[\mathrm{m}]}\end{array}$ & $\begin{array}{l}\hat{\mathbf{P}}^{\dagger} \\
{[\mathrm{m}]}\end{array}$ \\
\hline$T_{1}$ & $(-48,-90)$ & 10 & -8 & 533.5 & 637.6 & 0 & 0 & -7.99 & 9.96 & $(-45.5,-86.3)$ & $(-44.9,-85.2)$ & $(-45.2,-93.25)$ \\
\hline$T_{2}$ & $(-25,-80)$ & -2 & 20 & 673.7 & 413.4 & -1 & -1 & 19.86 & -1.57 & $(-32.6,36.8)$ & $(-31.4,-18.4)$ & $(-32.0,-78.8)$ \\
\hline$T_{3}$ & $(0,-75)$ & -6 & 16 & 939.9 & 727.7 & -1 & -1 & 15.90 & -5.65 & $(-5.9,46.3)$ & $(-4.7,-11.3)$ & $(-5.3,-72.5)$ \\
\hline$T_{4}$ & $(20,-70)$ & 15 & 4 & -267.3 & -319.3 & 0 & 0 & 4.00 & 15.86 & $(18.4,-75.1)$ & $(18.4,-76.3)$ & $(18.4,-71.7)$ \\
\hline$T_{5}$ & $(40,-70)$ & 20 & 2 & -115.1 & -175.2 & 0 & 0 & 1.98 & 20.28 & $(38.8,20.4)$ & $(39.7,21.3)$ & $(39.25,-76.15)$ \\
\hline$T_{6}$ & $(60,-65)$ & 25 & 5 & -331.3 & -397.4 & 0 & 0 & 4.97 & 24.82 & $(58.0,74.1)$ & $(57.7,74.9)$ & $(57.85,-69.0)$ \\
\hline
\end{tabular}

${ }^{\dagger} \mathrm{P}$ denotes the real position of the targets when $\eta=0 . \hat{P}_{1}$ and $\hat{P}_{2}$ denote the estimated position from the two SAR images generated by the pulse $c_{1}$ and pulse $c_{2} . \hat{P}$ is the final estimated result computed by equation (36). 

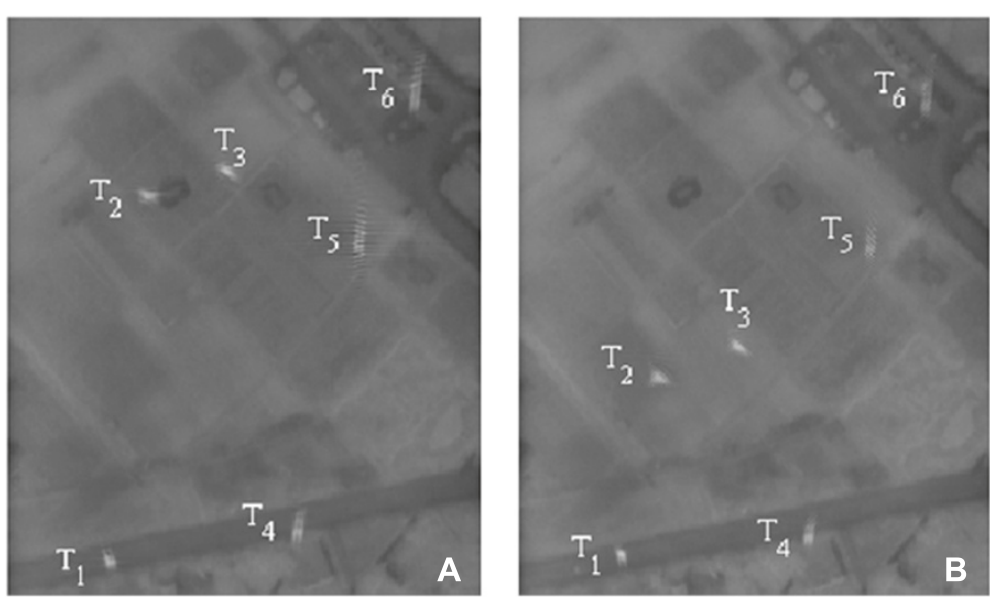

Figure 10 Computer simulated SAR images for $10 \mathrm{GHz}(A)$ and $12 \mathrm{GHz}$ (B).

The customized target size is $3 \mathrm{~m}$ in azimuth by $5 \mathrm{~m}$ in range with the average back reflective coefficient of the simulated scene. The targets are arranged to move on a road in the selected scene.

Figure 10A,B present two simulated SAR images generated by $c_{1}$ and $c_{2}$, respectively. In the two images, the background is well focused while the moving targets are smeared due to their motions. We defocused the images by using SDFPs corresponding to $\Delta V_{\mathrm{a}}=5 \mathrm{~m} / \mathrm{s}, 10 \mathrm{~m} / \mathrm{s}$, and $20 \mathrm{~m} / \mathrm{s}$. As a result, the moving targets are detected correctly.

The detected moving targets are isolated from the two original SAR images and the normalized Doppler difference curves of $T_{1} \sim T_{6}$ are computed. The results are
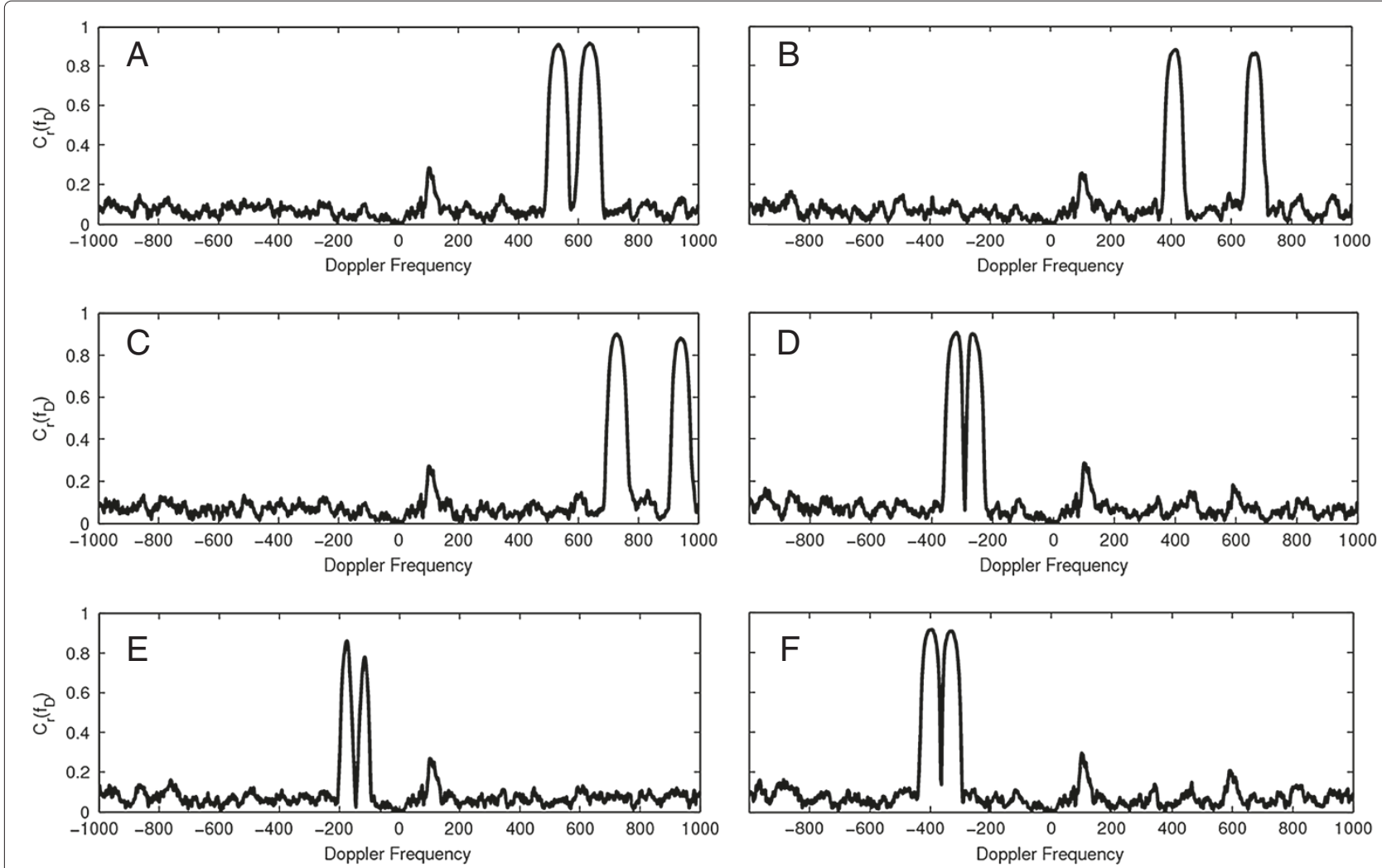

Figure 11 Normalized Doppler difference curves of six simulated moving targets. (A) presents the normalized Doppler difference of target $T_{1}$, (B) presents that of $\mathrm{T}_{2}, \mathbf{C}$ ) presents that of $\mathrm{T}_{3},(\mathbf{D})$ presents that of $\mathrm{T}_{4}, \mathbf{( E )}$ presents that of $\mathrm{T}_{5}$, and $(\mathbf{F})$ presents that of $\mathrm{T}_{6}$. 
presented in Figure 11A-F, respectively. The peak locations are listed in the columns $f_{\mathrm{dc} 1}$ and $f_{\mathrm{dc} 2}$ of Table 2 . The Doppler ambiguity is solved by the DAS algorithm and shown in the column of $\hat{m}$ and $\hat{n}$. The final range velocity components are computed by using the SAC algorithm. The results are listed in the column $\hat{V}_{\text {r }}$ of Table 2 . The estimated target positions are presented in the columns $\hat{P}_{1}$, $\hat{P}_{2}$, and $\hat{P}$ of Table 2 . The data in Table 2 confirm that the proposed DAS and SAC algorithms works well.

The detected targets are compensated by the range velocity estimates, and then the azimuth velocity components are computed then by constructing an SDFP bank with $\Delta V_{\mathrm{a}}$ ranging from 0 to $30 \mathrm{~m} / \mathrm{s}$ increased by the step $0.02 \mathrm{~m} / \mathrm{s}$. The sharpness difference curves of the moving targets have the same profiles as that presented in Figure 9. The estimated results are shown in the column $\hat{V}_{\mathrm{a}}$ of Table 2 . The results show that the estimate is not accurate when the azimuth velocity is less than $2 \mathrm{~m} / \mathrm{s}$. One principal reason lies in that the image of the moving target may be smeared slightly and thus the corresponding feature curve becomes so flat that the peak may appear at the position far from the real velocity value.

According to the estimates in the columns $\hat{V}_{\mathrm{r}}$ and $\hat{V}_{\mathrm{a}}$ of Table 2, the moving targets are refocused and their displaced azimuth positions are corrected. Without loss of generality, Figure 10A is selected as an example to show the correction and refocusing effectiveness. For each detected moving target in the SAR image, a slice which covers all the range bins that the moving target occupies is cropped. After range velocity compensated, it is refocused by using an azimuth matched filter with Doppler chirp rate $\hat{f}_{\mathrm{dr}}^{\mathrm{m}}=-2\left(V_{\mathrm{p}}-\hat{V}_{\mathrm{a}}\right)^{2} /\left(\lambda_{1} R_{0}\right)$. All the processed slices are stitched together with the original SAR image to show the real positions of the moving targets. Figure 12 presents the stitched product. From this figure we see that the moving targets are focused well while the background in each slice is smeared. The moving targets are just at the places that listed in column $\hat{P}^{\dagger}$ of Table 2.

\section{Conclusion}

A scheme for velocity components estimation in GMTI is proposed based on a single antenna SAR using a dualfrequency chirp waveform. The scheme consists of a moving target detector, a range velocity estimator and an azimuth velocity estimator, and it can locate the real position of the detected moving target by using a modified azimuth displacement correction model.

The moving target detector uses an SDFP to detect the possible moving targets with a limited azimuth velocity range near a certain value in the give complex SAR image. It is proved to be effective and efficient by field and simulated data. In practice, when the azimuth velocity varies widely, many SDFPs are needed to detect all the moving targets.

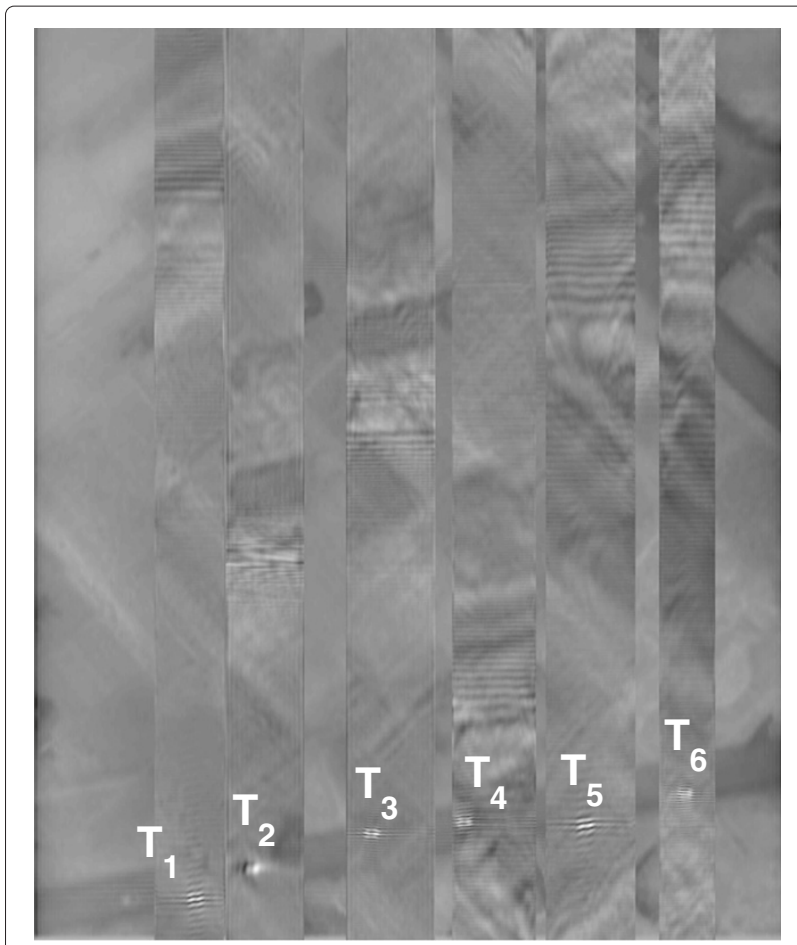

Figure 12 Refocused and azimuth-displacement-corrected moving targets.

The azimuth velocity estimator is established by using an SDFP bank. The effectiveness of the model is verified by simulated and field data. The AVE is robust because it tries to alleviate the influences of the clutters and interferences.

The range velocity estimator is based on the Doppler shift information introduced by range velocity component. Two algorithms, DAS and SAC, are designed to solve the Doppler ambiguity and make the Doppler centroid estimation more accurate. For each pulse burst, the baseband Doppler centroid is estimated by using a criterion named normalized Doppler spectrum difference. In addition, traditional azimuth displacement equation is modified to get the correct azimuth position of a moving target. Simulation results validate the improvement.

\section{Competing interests \\ The authors declare that they have no competing interests.}

\section{Acknowledgments}

We appreciate the support from the National Science Foundation of China (Grant Number: 61072150) and the National Basic Research Program of China (Grant Numbers: 2009CB824903 and 2010CB731904).

Received: 27 February 2012 Accepted: 25 September 2012

Published: 12 November 2012

\section{References}

1. PR Kersten, RW Jansen, K Luc, TL Ainsworth, Motion analysis in SAR images of unfocused objects using time-frequency methods. IEEE Geosci. Rem. Sens. Lett. 4(4), 527-531 (2007) 
2. $\mathrm{D} \mathrm{Vu}, \mathrm{B} \mathrm{Guo}, \mathrm{LXu}, \mathrm{Ji}$, in Algorithms for Synthetic Aperture Radar Imagery XVI, Proceedings of the SPIE. SAR based adaptive GMTI (vol. 7699, 76990H-76990H-12, Bellingham, WA, USA, 2010)

3. D Cerutti-Maori, CH Gierull, J Ender, Experimental verificaiton of SAR-GMT improvement through antenna switching. IEEE Trans. Geosci. Rem. Sens. 48(4), 2066-2075 (2010)

4. CH Gierull, Ground moving target parameter estimation for two-channe SAR. IET-Radar Sonar Navig. 153(3), 224-233 (2006)

5. G Krieger, N Gebert, A Moreira, Multidimensional waveform encoding: a new digital beamforming technique for synthetic aperture radar remote sensing. IEEE Trans. Geosci. Rem. Sens. 46, 31-46 (2008)

6. SK Wong, High range resolution profiles as motion-invariant features for moving ground targets identification in SAR-based automatic target recognition. IEEE Trans. Aerosp. Electron. Syst. 45(3), 1017-1039 (2009)

7. M Gisselfält, T Pernstål, in Proceedings of IEEE Radar Conference, vol. 2010 STAP analysis using multi-channel airborne radar data from flight trials (USA, Washington, DC, 2010), pp. 407-411

8. S Chiu, MV Dragošević, Moving target indication via RADARSAT-॥ multichannel synthetic aperture radar processing. EURASIP J. Adv. Signal Process. 2010(Article ID 740130), 1-19 (2010)

9. S Zhu, G Liao, Y Qu, Z Zhou, X Liu, Ground moving targets imaging algorithm for synthetic aperture radar. IEEE Trans. Geosci. Rem. Sens. 49, 462-477 (2011)

10. DM Vavriv, OO Bezvesilniy, in Proceedings of RAST, vol. 2011. Potential of multi-look SAR processing (Istanbul, Turkey, 2011), pp. 365-369

11. JR Fienup, Detecting moving targets in SAR imagery by focusing. IEEE Trans. Aerosp. Electron. Syst. 37(3), 794-809 (2001)

12. PAC Marques, JMB Dias, Moving targets processing in SAR spatial domain. IEEE Trans. Aerosp. Electron. Syst. 43(3), 864-874 (2007)

13. S Hinz, F Meyer, A Laika, R Bamler, in Proceedings of IEEE computer society conference CVPR. Spaceborne traffic monitoring with dual channel synthetic aperture radar-theory and experiments, vol. 13, 1063-6919/05 (San Diego, CA, USA, 2005). pp.1-7

14. DA Cook, DC Brown, Analysis of phase error effects on stripmap SAS. IEEE J. Ocean. Eng. 34(3), 250-261 (2009)

15. SV Baumgartner, G Krieger, Accleration-independent along-track velocity estimaiton of moving targets. IET-Radar Sonar Navig. 4(3), 474-487 (2009)

16. CY Chang, JC Curlander, Application of the multiple PRF technique to resolve Doppler centroid estimation ambiguity for spaceborne SAR. IEEE Trans. Geosci. Rem. Sens. 30(5), 941-949 (1992)

17. R Bamler, $H$ Runge, $P$ R F ambiguity resolving by wavelength diversity. IEEE Trans. Geosci. Rem. Sens. 29(6), 997-1003 (1991)

18. FH Wong, IG Cumming, A combined SAR Doppler centroid estimation scheme based upon signal phase. IEEE Trans. Geosci. Rem. Sens. 34(3) 696-717 (1996)

19. SN Madsen, Estimating the Doppler centroid of SAR data. IEEE Trans. Aerosp. Electron. Syst. 25(2), 134-140 (1989)

20. R Bamler, Doppler frequency estimation and the Cramer-Rao bound. IEEE Trans. Geosci. Rem. Sens. 29(3), 385-390 (1991)

21. JR Moreira, A new method of aircraft motion error extraction from radar raw data for real time motion compensation. IEEE Trans. Geosci. Rem. Sens. 28(4), 620-626 (1990)

22. JR Moreira, W Keydel, A new MTI-SAR approach using the reflectivity displacement method. IEEE Trans. Geosci. Rem. Sens. 33(5), 1238-1244 (1995)

23. J Wang, X Liu, in Proceedings of IEEE IGARSS, vol. 2011. Velocity estimation of moving targets using SAR (Vancouver, Canada, 2011), pp. 340-343

24. BC Wang, Digital Signal Processing Techniques and Applications in Radar Image Processing. (John Wiely and Sons Inc., Hoboken, 2008)

25. M Rüegg, E Meier, D Nüesch, Capabilities of dual-frequency millimeter wave SAR with monopulse processing for ground moving target indication. IEEE Trans. Geosci. Rem. Sens. 45(3), 539-553 (2007)

26. RK Raney, Synthetic aperture imaging radar and moving targets. IEEE Trans. Aerosp. Electron. Syst. AES-7(3), 499-505 (1971)
27. IG Cumming, S Li, Improved slope estimation for SAR Doppler ambiguity resolution. IEEE Trans. Geosci. Rem. Sens. 44(3), 707-718 (2006)

28. JC Lagarias, JA Reeds, MH Wright, PE Wright, Convergence properties of the Nelder-Mead simplex method in low dimensions. SIAM J. Optim. 9 112-147 (1998)

29. O Dogan, M Kartal, Efficient strip-mode SAR raw-data simulation of fixed and moving targets. IEEE Geosci. Rem. Sens. Lett. 8(5), 884-888 (2011)

doi:10.1186/1687-6180-2012-236

Cite this article as: Lv et al.: Ground moving target indication and parameters estimation using a dual-frequency synthetic aperture radar. EURASIP Journal on Advances in Signal Processing 2012 2012:236.

\section{Submit your manuscript to a SpringerOpen ${ }^{\circ}$ journal and benefit from:}

- Convenient online submission

- Rigorous peer review

- Immediate publication on acceptance

- Open access: articles freely available online

- High visibility within the field

- Retaining the copyright to your article

Submit your next manuscript at $\boldsymbol{\nabla}$ springeropen.com 